\title{
Critical review of the responsive neurostimulator system for epilepsy
}

This article was published in the following Dove Press journal:

Medical Devices: Evidence and Research

I October 2015

Number of times this article has been viewed

\section{George P Thomas \\ Barbara $C$ Jobst}

Dartmouth-Hitchcock Medical Center, Geisel School of Medicine, Dartmouth College, Lebanon, NH, USA
Correspondence: Barbara C Jobst Dartmouth-Hitchcock Medical Center, Geisel School of Medicine, Dartmouth College, One Medical Center Drive, Lebanon, NH 03756, USA
Abstract: Patients with medically refractory epilepsy have historically had few effective treatment options. Electrical brain stimulation for seizures has been studied for decades and ongoing technological refinements have made possible the development of an implantable electrical brain stimulator. The NeuroPace responsive neurostimulator was recently approved by the FDA for clinical use and the initial reports are encouraging. This device continually monitors brain activity and delivers an electric stimulus when abnormal activity is detected. Early reports of efficacy suggest that the device is well tolerated and offers a reduction in seizure frequency by approximately half at 2 years.

Keywords: medically refractory epilepsy, seizures, brain surgery, brain stimulation, neurostimulation

\section{Introduction}

Epilepsy affects approximately $1 \%$ of the population, with between $30 \%$ and $40 \%$ of these patients suffering from drug-resistant disease. Epilepsy surgery historically has been one of the options for such refractory disease and on average results in $62 \%$ of all patients becoming seizure-free. ${ }^{1}$ However, epilepsy surgery is not without inherent risks and sometimes is not possible due to a resulting neurologic deficit. Therefore, there has been a long-standing need for other treatment options for drug-resistant epilepsy.

Penfield and Jasper were the first to report the effects of electrical stimulation on electrocorticogram (ECoG) activity. ${ }^{2}$ Over the following decades, numerous descriptions of electrical brain stimulation for seizures were reported, targeting different anatomic regions and using various modes of scheduled stimulation. Early studies focused on stimulation of regions with widespread inhibitory output such as the cerebellum, ${ }^{3-6}$ but the effects of stimulation of other regions including the hippocampus, ${ }^{7,8}$ anterior thalamic nucleus, ${ }^{9}$ and cortex ${ }^{10,11}$ have been reported.

Most of these studies included only small cohorts without control groups. To date, there has been only one large randomized controlled study of scheduled deep brain stimulation performed in the US. ${ }^{12}$ Patients with focal epilepsy received high-frequency stimulation of the bilateral anterior nucleus of the thalamus (1 minute on, 5 minutes off) with a device also used for stimulation of the subthalamic nucleus in Parkinson's disease (Model 7428 Kinetra Neurostimulator; Medtronic, Inc., Minneapolis, MN, USA). In the study period, patients had a $40.5 \%$ reduction in seizure frequency compared with a $14.5 \%$ reduction in the control group. This device is not approved in the US for epilepsy but is available in Europe. 
Vagus nerve stimulation (VNS) as a means of indirect brain stimulation has been used for seizure control, ${ }^{13-15}$ and a recent Cochran Review summarizes that VNS is effective and well tolerated. ${ }^{16}$ VNS has also been used for the treatment of chronic depression ${ }^{17}$ and a recent report describes percutaneous VNS for weight loss. ${ }^{18}$ This modality therefore appears to hold promise for a variety of conditions, and new less invasive variations might offer increased accessibility to a wider spectrum of patients.

Currently, in the US, the only approved device for direct electrical brain stimulation for epilepsy is the responsive neurostimulator system (RNS, NeuroPace, Inc., Mountainview, CA, USA). This device is approved for partial-onset, medically refractory epilepsy. In contrast to scheduled or continuous stimulation, the RNS delivers electrical stimulation in response to real-time ECoG activity. The aim of this paper is to serve as an introduction to the principles of responsive brain stimulation and describe the indications, basis, safety, and efficacy of the currently available responsive neurostimulator (RNS).

\section{The case for responsive electrical brain stimulation for epilepsy}

Electrical brain stimulation has been found to be an important adjunctive treatment in advanced Parkinson's disease. ${ }^{19}$ In this setting, stimulation is delivered on a scheduled basis, without modulation based on the electrical activity of the target tissue. This "open-loop" mode of stimulation, which is effective in movement disorders, has been employed for epilepsy in the anterior thalamic nucleus for seizures of focal onset. The anterior thalamic nucleus is an integral part of the limbic circuit and is therefore thought to influence seizure threshold. This mode of stimulation reduces seizure frequency with the stimulator on as compared with off. ${ }^{12}$ There have been some concerns about the development of depression with this mode of stimulation; ${ }^{20}$ however, long-term follow up has not supported this. ${ }^{21}$ An alternative approach is the delivery of intermittent stimulation in response to epileptiform activity with the aim of preventing or limiting abnormal electrical activity from propagating into a clinical seizure.

\section{The RNS system}

The RNS system is currently approved in the USA as an adjunctive therapy for medically resistant partial-onset seizures. The RNS system consists of a stimulator, implanted leads, and a wireless programming wand with associated computer hardware and software. The stimulator containing the batteries, circuitry, and radio for interrogation and programming is implanted in a burr hole in the patient's skull. The stimulator is connected via thin flexible wires to electrodes that may be either implanted in deep structures such as the hippocampus or placed on the surface of the brain (Figure 1). The target for stimulation is typically the seizure onset zone(s). The stimulation electrodes can be placed in alternate hemispheres as with bitemporal epilepsy or epilepsy with two seizure onset zones. Each electrode has four contacts that can be programmed as either anode or cathode; alternatively, the stimulator case can be programmed to serve as the cathode. (Figure 2).

\section{Seizure detection}

The neurostimulator continuously monitors ECoG activity and uses several methods, or tools, to detect abnormal electrical activity. The area tool measures changes in overall EEG signal intensity, the line length tool monitors dynamic changes in electrical activity and frequency, and the halfwave tool is designed to detect spike discharged and activity within specific frequency bands. The parameters of these
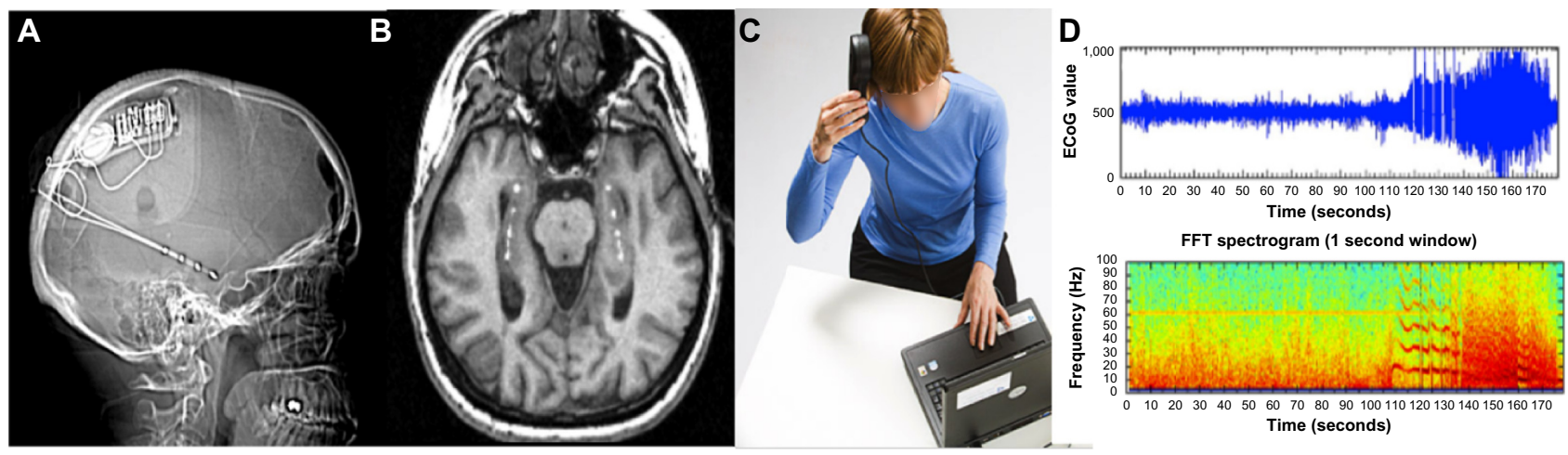

Figure I Components and programming of the NeuroPace RNS.

Notes: (A) The RNS on skull X-ray. (B) Example of a bilateral hippocampal implantation. (C) Interrogation of the device with the wireless wand and the programmer. (D) ECoG and time-frequency analysis from the device with detection of an epileptic seizure and stimulation delivered (vertical lines).

Abbreviations: ECoG, electrocorticogram; FFT, fast Fourier transform; RNS, responsive neurostimulator. 


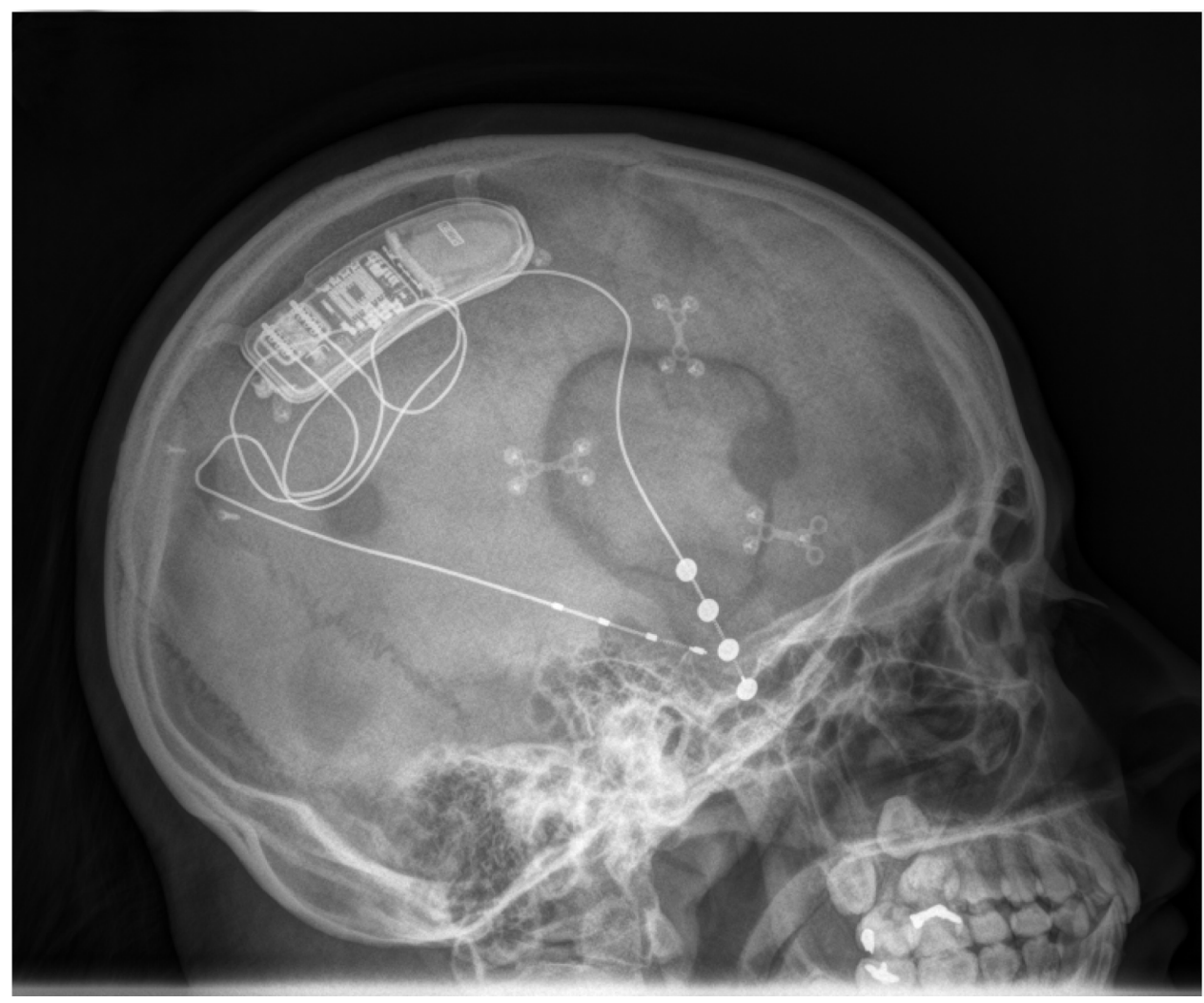

Figure 2 RNS stimulator with depth and surface electrodes.

Notes: Example of a patient with both neocortical surface electrodes and hippocampal depth electrodes.

Abbreviation: RNS, responsive neurostimulator.

tools can be modified by the clinician to improve sensitivity and specificity of detection of abnormal ECoG activity in individual patients.

When any of the detection tools sense abnormal activity, a current-controlled, charge balanced biphasic electrical stimulation is delivered to the brain. The frequency of stimulation can be adjusted from $1 \mathrm{~Hz}$ up to $333 \mathrm{~Hz}$, and currents ranging from $1 \mathrm{~mA}$ to $12 \mathrm{~mA}$ are available. The pulse width of stimulation can be adjusted between $40 \mu \mathrm{s}$ and 1,000 $\mu \mathrm{s}$. Stimulation can be delivered between any two of the electrodes, or between any electrode and the neurostimulator case. Current densities are maintained $<25 \mathrm{uC} / \mathrm{cm}^{2}$ and usually are much lower than these safe limits. ${ }^{22}$ Typical stimulation parameters applied in clinical practice are a current of 1.5-3 mA, pulse width of $160 \mu \mathrm{s}$, pulse duration of $100-200 \mathrm{~ms}$, and frequency of 100-200 Hz. While detections and stimulations are frequent throughout the day, the total current density delivered remains low compared with deep brain stimulation.

\section{Initial studies of safety and efficacy}

The initial report describing the safety and efficacy of the NeuroPace device in 191 subjects was published in 2011. Patients with medically refractory partial-onset epilepsy were randomized to the stimulation or control group 2 months after implantation. ${ }^{23}$ Initially, all patients had a reduction in seizure frequency right after implantation (an effect noted after implantation of any device and now termed the "implantation effect"12,23). Those in the treatment arm $(n=97)$ showed a $37.9 \%$ decrease in self-reported seizures over 12 weeks, while those in the nontreatment arm $(n=94)$ showed a $17.3 \%$ decrease over the same time period (generalized estimation equation analysis, $P<0.01$ ). During a subsequent open-label period where all subjects received responsive stimulation, the improvement in the treatment group continued, and the sham group exhibited a decrease in seizure frequency similar to that seen in the treatment group.

Long-term, noncontrolled studies found that self-reported seizures were reduced by $44 \%$ at 1 year and $53 \%$ at 2 years after implantation. ${ }^{24}$ Further seizure reduction ranging between $48 \%$ and $66 \%$ was observed over years 3 through 6.25 Adverse events during the 1 st year included implant site pain (15.7\%), headache (10.5\%), and dysesthesias (6.3\%) and were considered comparable with those seen with similar procedures. ${ }^{23}$ The most common long-term complications reported with the NeuroPace device were implant site infection $(9.0 \%)$ and stimulator explanation $(4.7 \%) .{ }^{25}$ 

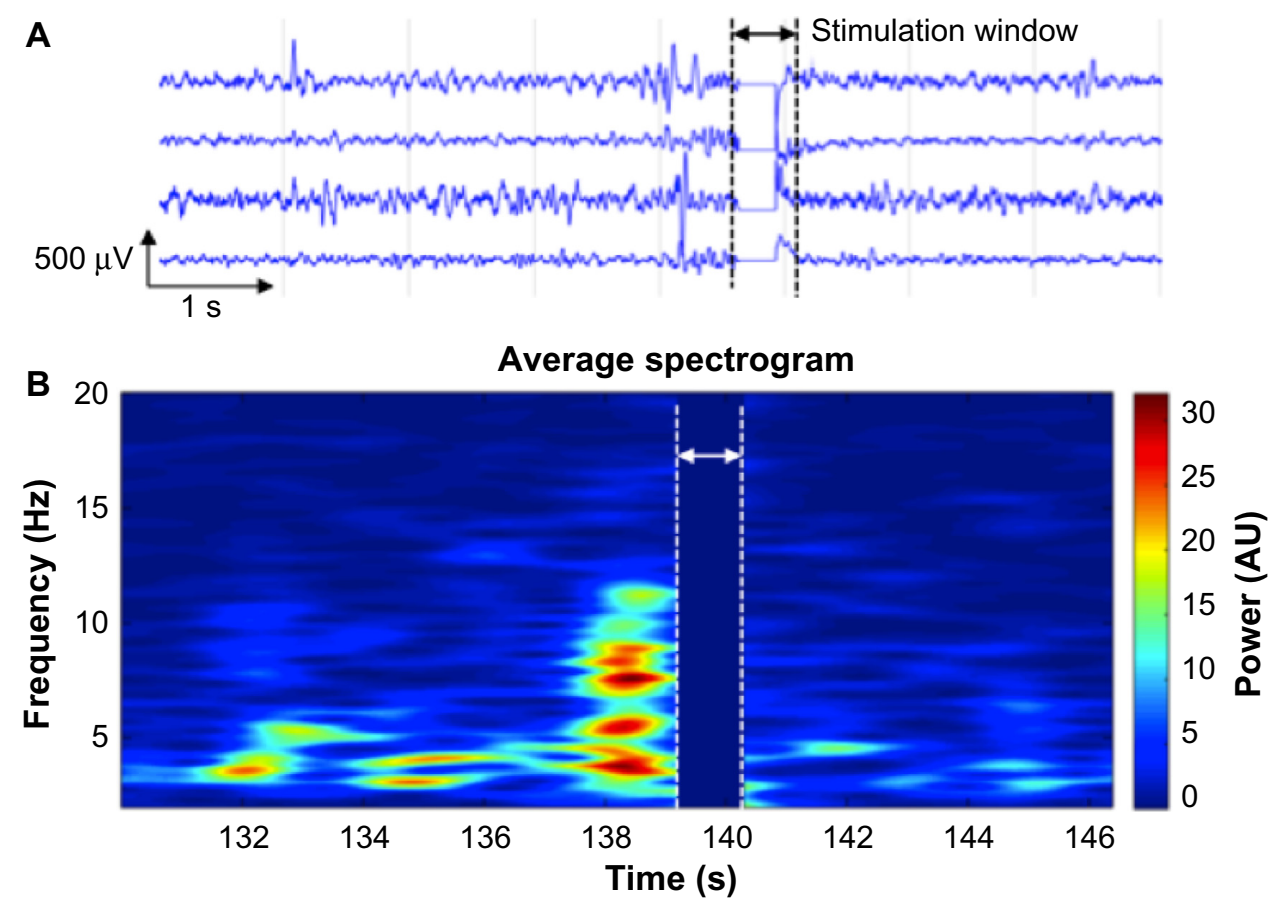

Figure 3 RNS ECoG recorded during stimulation.

Notes: Stimulation is triggered by epileptiform activity in recorded channels (A). The recording is turned off during stimulus. The average spectrogram (B) shows strongly reduced spectral power after stimulation.

Abbreviations: AU, arbitrary units; ECoG, electrocorticogram; RNS, responsive neurostimulator.

\section{Effects of the RNS on depression, cognition, and quality of life}

Over a follow-up period of up to 7 years, closed-loop stimulation with the RNS does not appear to have a detrimental effect on mood. ${ }^{25}$ Quality of life is another important metric used to characterize the effects of epilepsy treatment. Using the QOLIE-89 assessment, ${ }^{26}$ participants showed an increase in scores at 5 years. ${ }^{25}$

\section{Mortality}

The RNS Study Group reported a total of eleven deaths in their cohort over 7 years of observation. Seven of these were attributed to SUDEP (possible, probably, or definite), two participants with a history of depression died by committing suicide, one participant with subtherapeutic antiepileptic drug levels died of status epilepticus, and one subject died from lymphoma. ${ }^{25}$ Although the incidence of SUDEP varies by age and sex, published estimates indicate an incidence of 1.21 deaths/1,000 patients/year. ${ }^{27}$ Based on the initial sample size over the course of 7 years, the expected number of deaths attributed to SUDEP in the RNS Study Group is approximately 0.9 and consistent with what is expected in this very refractory epilepsy population.

One of the earliest criticisms of the RNS studies was the reliance on self-reporting of seizures particularly given that the device is capable of recording eletrocorticography that could allow precise assessment of the number and nature of electrical abnormalities. ${ }^{28}$ The RNS system in its current form, however, does not allow for extended recordings and only allows for limited sampling for select ECoGs. A complete record of this data over an extended duration is therefore not feasible, and self-reporting of seizures was the only viable method to track efficacy. It was also noted that despite the potential for bias and error, self-reporting of seizure frequency is a commonly used method of data collection in epilepsy studies. ${ }^{29}$ The limitations of the general estimating equation method used in the statistical analysis in accounting for variation in seizure pattern have also been criticized. ${ }^{28}$ While this statistical method might not be able to capture changes in seizure patterns, patients do report fewer seizures with responsive neurostimulation and the device appears to be well tolerated.

\section{Mechanisms of action}

The mechanism mediating the effects of electrical brain stimulation on seizures is not clear, but there are probably multiple short and long-term effects. For comparison, four distinct effects on neural activity have been postulated to account for the effects of DBS on Parkinsonism: depolarization blockade refers to changes brought about at voltage-gated ion 
channels leading to local inhibition of excitability, synaptic inhibition refers to the effects of depolarization of the distal axon directly by electrical stimulation, synaptic depression occurs when the electrical stimulation leads to depletion of neurotransmitter at the axon terminal, ${ }^{30}$ and lastly, electrical stimulation might through any of the prior means modulate the activity of pathologic networks. ${ }^{31}$

\section{Acute effects of electrical brain stimulation}

Some of the acute changes seen with electrical brain stimulation of epileptogenic regions appear to include disruption of synchronous activity and evolution into generalized seizures. ${ }^{32-34}$ Electrical stimulation also appears to influence short-term activity distant to the site of stimulation, modulating function at the network level. ${ }^{35,36}$ There is some evidence that these acute effects of cortical stimulation might be related to changes in GABA-mediated hyperpolarization. High-frequency stimulation $>100 \mathrm{~Hz}$ appears to up-regulate glutamic acid decarboxylase while down-regulating calmodulin-dependent protein kinase II and thereby lead to local inhibition. ${ }^{37}$ Repetitive electrical stimulation of CA3 mossy fibers leads to increased intracellular chloride, ${ }^{38-40}$ also consistent with a GABA-mediated mechanism. Thus, it appears that electrical stimulation has an acute, local inhibitory effect (Figure 3).

\section{Long-term effects of electrical brain stimulation}

The progressive reduction in seizure frequency over time ${ }^{25}$ suggests that electrical stimulation might have effects beyond those on ion channels. Again drawing from the field of deep brain stimulation for movement disorders, Wistar rats undergoing 2 hours of high-frequency deep brain stimulation directed at the globus pallidus interna exhibited a threefold increase in glial cell-line-derived (GDNF) alpha-1-a mRNA expression, without altering the expression of GDNF-alpha1-b mRNA. GDNF is known to be neuroprotective to dopaminergic neurons, and these results suggest that part of the beneficial effects of deep brain stimulation for Parkinsonism might be related to modulation of neurotrophic factors at the site of stimulation. ${ }^{41}$ Furthermore, such modulation of gene expression might not be spatially limited to the area of stimulation. Hooded Lister rats undergoing 3 hours of highfrequency $(130 \mathrm{~Hz})$ stimulation of the mediodorsal thalamic nucleus exhibited increased expression of zif-268 but not c-fos in prelimbic, orbitofrontal, and premotor cortex regions known to receive input from the mediodorsal thalamus. ${ }^{42}$ It therefore appears that some of the long-term effects of brain stimulation on movement disorders might be related to modulation of genetic expression both locally and in regions receiving projections from the area undergoing stimulation. Based on these observations and reports of continual improvement in seizure frequency over months of stimulation, it seems that modulation of gene expression might underlie some to the effects of brain stimulation for epilepsy.

\section{Other effects of long-term brain implantation}

Again drawing from experience with deep brain stimulation for movement disorders, there appears to be a low rate of acute complications that include mechanical failure $(3.1 \%)$, hemorrhage or infarction $(1.2 \%)$, and infection $(0.4 \%)$. Similar rates and types of complications have been reported with electrodes placed for brain stimulation in epilepsy. ${ }^{23,43}$

Implantation of stimulation electrodes is known to produce both acute and chronic immunologic reactions that might affect performance over time. An astrocytic reaction is seen immediately after implantation, followed by the formation of gliosis surrounding the electrodes. ${ }^{44}$ The size and shape of the electrode, as well as any movement after implantation, appear to influence the development of this glial scar. This gliosis can alter electrical impedances and therefore degrade the effectiveness of stimulation over time. ${ }^{45,46}$ Changes in impedance over time have been examined as it appears that there are variations over several weeks postimplantation particularly with subdural electrodes. ${ }^{46}$ Electrical impedance appears to stabilize after 1 year, however, and the constant current design of the RNS compensates for changes in impedance and maintaining performance over time. ${ }^{45}$

\section{Conclusion}

The NeuroPace RNS reflects the culmination of several decades of research in electrical stimulation for seizures and offers an alternative to traditional epilepsy surgery for patients with drug-resistant partial-onset seizures. Initial reports describe substantial reductions in seizure frequency, with improvements in measures of quality of life, as well as no negative effects on mood or depression.

There likely are multiple mechanisms mediating the effects of electrical brain stimulation on seizures. In the short term, influences on GABA-mediated hyperpolarization might account for the effects of stimulation on seizure generation and propagation. The observation that seizure frequency continues to decrease over time suggests that electrical stimulation might alter gene expression or perhaps brain network architecture and connectivity. 
Although initial reports of efficacy are promising, the RNS device might not be as effective in seizure reduction as epilepsy surgery. Further improvements in detection and stimulation paradigms are under investigation. The rates of the most frequently encountered risks of infection and explantation appear to be similar to the rates to comparable procedures.

The RNS represents a milestone in the treatment of medically resistant epilepsy, providing an alternative to surgery that is both adjustable and reversible. As currently used, the RNS appears to be effective and well tolerated and has the capability to treat epilepsy syndromes that had no further treatment options such as bitemporal epilepsy or epilepsy with onset in the eloquent areas of the brain. Further research and clinical experience with this new treatment will provide a more clear understanding of the mechanisms underlying its effect on seizures, as well as further refinement of indications and applications.

\section{Disclosure}

George P Thomas is a reviewer for Barnes and Jones Publishers. Barbara C Jobst reports research support from NeuroPace, Inc., Lundbeck, Inc., the American Epilepsy Society, the Diamond Foundation, the CDC (grant numbers U48DP005018 and 3U48DP001935-04S3), the NIH (grant number 5R01NS074450-02), and DARPA (Prime Award N66001-14-2-4-31); received honoraria for: "Cognitive and behavioral consequences in epilepsy" from Helle ecole J Bancaud 2015; "One Step Forward: Brain Stimulation and Cognition in Epilepsy" from the University of Wisconsin, Neurology Grand Rounds, and the University of Massachusetts, Neurology Grand Rounds; and for "Seizure semiology: Clues for Localization and Lateralization", from the Mayo Clinic, Neuroscience Grand Rounds. Barbara C Jobst also reports involvement with the Neurology Advisory Board of NeuroPace, Inc., and a role as Editor for Epilepsia, Epilepsy Currents. The authors report no other conflicts of interest in this work.

\section{References}

1. Jobst BC, Cascino GD. Resective epilepsy surgery for drug-resistant focal epilepsy: a review. JAMA. 2015;313(3):285-293.

2. Wilder P, Herbert J. Epilepsy and the Functional Anatomy of the Human Brain. Boston, MA: Little, Brown; 1954.

3. Cooper IS, Amin I, Riklan M, Waltz JM, Poon TP. Chronic cerebellar stimulation in epilepsy. Clinical and anatomical studies. Arch Neurol. 1976;33(8):559-570.

4. Cooper IS, Amin I, Upton A, Riklan M, Watkins S, McLellan L. Safety and efficacy of chronic cerebellar stimulation. Appl Neurophysiol. 1977;40(2-4):124-134.
5. Rosenow J, Das K, Rovit RL, Couldwell WT, Irving S. Cooper and his role in intracranial stimulation for movement disorders and epilepsy. Stereotact Funct Neurosurg. 2002;78(2):95-112.

6. Van Buren JM, Wood JH, Oakley J, Hambrecht F. Preliminary evaluation of cerebellar stimulation by double-blind stimulation and biological criteria in the treatment of epilepsy. J Neurosurg. 1978;48: 407-416.

7. Velasco AL, Velasco F, Velasco M, Trejo D, Castro G, Carrillo-Ruiz JD. Electrical stimulation of the hippocampal epileptic foci for seizure control: a double-blind, long-term follow-up study. Epilepsia. 2007; 48(10):1895-1903.

8. Boon P, Vonck K, De Herdt V, et al. Deep brain stimulation in patients with refractory temporal lobe epilepsy. Epilepsia. 2007;48(8): $1551-1560$.

9. Velasco F, Velasco M, Ogarrio C, Fanghanel G. Electrical stimulation of the centromedian thalamic nucleus in the treatment of convulsive seizures: a preliminary report. Epilepsia. 1987;28(4):421-430.

10. Kinoshita M, Ikeda A, Matsuhashi M, et al. Electric cortical stimulation suppresses epileptic and background activities in neocortical epilepsy and mesial temporal lobe epilepsy. Clin Neurophysiol. 2005; 116(6):1291-1299.

11. Kinoshita M, Ikeda A, Matsumoto R, et al. Electric stimulation on human cortex suppresses fast cortical activity and epileptic spikes. Epilepsia. 2004;45(7):787-791.

12. Fisher R, Salanova V, Witt T, et al; SANTE Study Group. Electrical stimulation of the anterior nucleus of thalamus for treatment of refractory epilepsy. Epilepsia. 2010;51(5):899-908.

13. Ben-Menachem E, Mañon-Espaillat R, Ristanovic R, et al. Vagus nerve stimulation for treatment of partial seizures: 1. A controlled study of effect on seizures. First International Vagus Nerve Stimulation Study Group. Epilepsia. 1994;35(3):616-626.

14. Michael JE, Wegener K, Barnes DW. Vagus nerve stimulation for intractable seizures: one year follow-up. J Neurosci Nurs. 1993;25(6): 362-366.

15. Salinsky MC, Burchiel KJ. Vagus nerve stimulation has no effect on awake EEG rhythms in humans. Epilepsia. 1993;34(2):299-304.

16. Panebianco M, Rigby A, Weston J, Marson AG. Vagus nerve stimulation for partial seizures. Cochrane Database Syst Rev. 2015;4: CD002896.

17. Ben-Menachem E, Revesz D, Simon BJ, Silberstein S. Surgically implanted and non-invasive vagus nerve stimulation: a review of efficacy, safety and tolerability. Eur J Neurol. 2015;22(9):1260-1268.

18. Kassir R, Barthelemy JC, Roche F, et al. Bariatric surgery associated with percutaneous auricular vagal stimulation: a new prospective treatment on weight loss. Int J Surg. 2015;18:55-56.

19. Deuschl G, Paschen S, Witt K. Clinical outcome of deep brain stimulation for Parkinson's disease. Handb Clin Neurol. 2013;116:107-128.

20. Nowell M, Miserocchi A, McEvoy AW, Duncan JS. Advances in epilepsy surgery. J Neurol Neurosurg Psychiatry. 2014;85(11):1273-1279.

21. Salanova V, Witt T, Worth R, et al; SANTE Study Group. Long-term efficacy and safety of thalamic stimulation for drug-resistant partial epilepsy. Neurology. 2015;84(10):1017-1025.

22. Sun FT, Morrell MJ. Closed-loop neurostimulation: the clinical experience. Neurotherapeutics. 2014;11(3):553-563.

23. Morrell MJ. Responsive cortical stimulation for the treatment of medically intractable partial epilepsy. Neurology. 2011;77(13): 1295-1304.

24. Heck CN, King-Stephens D, Massey AD, et al. Two-year seizure reduction in adults with medically intractable partial onset epilepsy treated with responsive neurostimulation: final results of the RNS system pivotal trial. Epilepsia. 2014;55(3):432-441.

25. Bergey GK, Morrell MJ, Mizrahi EM, et al. Long-term treatment with responsive brain stimulation in adults with refractory partial seizures. Neurology. 2015;84(8):810-817.

26. Devinsky O, Vickrey BG, Cramer J, et al. Development of the quality of life in epilepsy inventory. Epilepsia. 1995;36(11):1089-1104. 
27. Walczak TS, Leppik IE, D'Amelio M, et al. Incidence and risk factors in sudden unexpected death in epilepsy: a prospective cohort study. Neurology. 2001;56(4):519-525.

28. Osorio I. The NeuroPace trial: missing knowledge and insights. Epilepsia. 2014;55(9):1469-1470.

29. Morrell MJ. In response: the RNS system multicenter randomized double-blinded controlled trial of responsive cortical stimulation for adjunctive treatment of intractable partial epilepsy: knowledge and insights gained. Epilepsia. 2014;55(9):1470-1471.

30. Beurrier C, Bioulac B, Audin J, Hammond C. High-frequency stimulation produces a transient blockade of voltage-gated currents in subthalamic neurons. J Neurophysiol. 2001;85(4):1351-1356.

31. McIntyre CC, Savasta M, Kerkerian-Le Goff L, Vitek JL. Uncovering the mechanism(s) of action of deep brain stimulation: activation, inhibition, or both. Clin Neurophysiol. 2004;115(6):1239-1248.

32. Sohal VS, Sun FT. Responsive neurostimulation suppresses synchronized cortical rhythms in patients with epilepsy. Neurosurg Clin NAm. 2011;22:481-488

33. Durand D. Electrical stimulation can inhibit synchronized neuronal activity. Brain Res. 1986;382(1):139-144.

34. Khosravani H, Carlen PL, Velazquez JL. The control of seizure-like activity in the rat hippocampal slice. Biophys J. 2003;84(1):687-695.

35. D'Arcangelo G, Panuccio G, Tancredi V, Avoli M. Repetitive lowfrequency stimulation reduces epileptiform synchronization in limbic neuronal networks. Neurobiol Dis. 2005;19(1-2):119-128.

36. Osorio I, Frei MG, Sunderam S, et al. Automated seizure abatement in humans using electrical stimulation. Ann Neurol. 2005;57(2): 258-268.

37. Liang F, Isackson PJ, Jones EG. Stimulus-dependent, reciprocal up- and downregulation of glutamic acid decarboxylase and $\mathrm{Ca} 2+/$ calmodulindependent protein kinase II gene expression in rat cerebral cortex. Exp Brain Res. 1996;110(2):163-174.
38. Thompson SM, Gahwiler BH. Activity-dependent disinhibition. II. Effects of extracellular potassium, furosemide, and membrane potential on ECl- in hippocampal CA3 neurons. $J$ Neurophysiol. 1989;61(3):512-523.

39. Thompson SM, Gahwiler BH. Activity-dependent disinhibition. I. Repetitive stimulation reduces IPSP driving force and conductance in the hippocampus in vitro. J Neurophysiol. 1989;61(3):501-511.

40. Thompson SM, Gahwiler BH. Activity-dependent disinhibition. III. Desensitization and GABAB receptor-mediated presynaptic inhibition in the hippocampus in vitro. J Neurophysiol. 1989;61(3):524-533.

41. Ho DX, Tan YC, Tan J, Too HP, Ng WH. High-frequency stimulation of the globus pallidus interna nucleus modulates GFRalphal gene expression in the basal ganglia. J Clin Neurosci. 2014;21(4):657-660.

42. Ewing SG, Porr B, Pratt JA. Deep brain stimulation of the mediodorsal thalamic nucleus yields increases in the expression of zif-268 but not c-fos in the frontal cortex. J Chem Neuroanat. 2013;52:20-24.

43. Cook MJ, O'Brien TJ, Berkovic SF, et al. Prediction of seizure likelihood with a long-term, implanted seizure advisory system in patients with drug-resistant epilepsy: a first-in-man study. Lancet Neurol. 2013; 12(6):563-571.

44. Polikov VS, Tresco PA, Reichert WM. Response of brain tissue to chronically implanted neural electrodes. JNeurosci Methods. 2005;148 $1-18$.

45. Sillay KA, Rutecki P, Cicora K, et al. Long-term measurement of impedance in chronically implanted depth and subdural electrodes during responsive neurostimulation in humans. Brain Stimul. 2013; 6(5):718-726.

46. Wu C, Evans JJ, Skidmore C, Sperling MR, Sharan AD. Impedance variations over time for a closed-loop neurostimulation device: early experience with chronically implanted electrodes. Neuromodulation. 2013;16(1):46-50.
Medical Devices: Evidence and Research

\section{Publish your work in this journal}

Medical Devices: Evidence and Research is an international, peerreviewed, open access journal that focuses on the evidence, technology, research, and expert opinion supporting the use and application of medical devices in the diagnosis, treatment and management of clinical conditions and physiological processes. The identification of novel

\section{Dovepress}

devices and optimal use of existing devices which will lead to improved clinical outcomes and more effective patient management and safety is a key feature. The manuscript management system is completely online and includes a quick and fair peer-review system. Visit http://www. dovepress.com/testimonials.php to read real quotes from authors. 